\title{
Morbid Adherent Placenta among Pregnant Women prior to Cesarean Delivery: Experience of 15 Cases at a Tertiary care Hospital in Bangladesh
}

\author{
Tahamina Khanum¹, Gulshan Ara², Sheuly Begum³, Mohammed Zubayer Miah', \\ Shimul Akter ${ }^{5}$
}

\begin{abstract}
${ }^{1}$ Assistant Professor, Department of Gynaecology \& Obstetrics, Enam Medical College, Dhaka, Bangladesh; ${ }^{2}$ Professor \& Head, Department of Gynaecology \& Obstetrics, Enam Medical College, Dhaka, Bangladesh; ${ }^{3}$ Associate Professor, Department of Gynaecology \& Obstetrics, Enam Medical College, Dhaka, Bangladesh; ${ }^{4}$ Assistant Professor, Department Of Psychiatry, Shaheed Tajuddin Ahmad Medical College, Gazipur, Bangladesh; ${ }^{5}$ Assistant Professor, Department of Gynaecology and Obstetrics, Monowara Sikder Medical College, Shariatpur, Bangladesh
\end{abstract}

[Received: 12 November 2019; Accepted: 2 December 2019; Published: 1 January 2020]

\begin{abstract}
Background: Morbid adherent placenta among pregnant women is a very serious condition. Objective: The purpose of the present study was to identify the risk factors predisposing to morbidly adherent placenta, different modes of management and maternal and neonatal outcomes. Methodology: This cohort study was conducted in the Department of Obstetrics and Gynaecology in Enam Medical College, Dhaka, Bangladesh from January to December 2018 for a period of one (01) year. The study included all pregnant women, irrespective of parity status, with gestational age more than 29 weeks who had any type of morbid adherent placenta. The diagnosis was made only during attempted manual removal when the plane of cleavage between the placenta and the uterine wall could not be made out. The complete history, physical examination of the patient along with relevant investigations for diagnosis of morbid adherent placenta were documented. The mood of the presentation of the patient and duration of gestation, history of previous lower segment or classical caesarean section or other intrauterine operative procedure, by lateral uterine ligation, internal iliac artery ligation, hysterectomy-classical or lower segment or peri-partum, neonatal outcome and duration of the hospital stay all were recorded. Result: The incidence of morbidly adherent placenta was 12.75 per 1000 pregnancies. Patient profile comprising third gravida in the age group of 25 to 28 years, $92 \%$ of the patient in this study have previous caesarean section and co-existing placenta praevia was diagnosed in $93 \% .47 \%$ of the women delivered between $36 \& 38$ weeks. Caesarean section was the mode of delivery in $93 \%$ of the patient. Conclusion: In conclusion young adult women with previous caesarean section with co-existenting of placenta praevia are the most commonly suffering from morbidly adherent placenta. [Journal of National Institute of Neurosciences Bangladesh, 2020;6(1): 33-37]
\end{abstract}

Keywords: : Morbid adherent placenta; pregnant women; placenta accrete; placenta increta; myometrium

Correspondence: Dr. Tahamina Khanum, Assistant Professor, Department of Gynaecology \& Obstetrics, Enam Medical College, Savar, Dhaka, Bangladesh; Cell no.:+8801711934097; Email: drtahamina75@gmail.com

Conflict of interest: There is no financial conflict of interest relevant to this paper to disclose.

Funding agency: This project was not funded by any group, organization or institution.

Contribution to authors: Khanum T, Ara G, were involved in concept and design of the study; however, Khanum T was involved in data collection, data compilation, processing and analysis, while Begum S, Miah MZ, Akter S were equally involved in the literature search, compilation, manuscript writing and revision.

How to cite this article: Khanum T, Ara G, Begum S, Miah MZ, Akter S. Morbid Adherent Placenta among Pregnant Women prior to Cesarean Delivery: Experience of 15 Cases at a Tertiary care Hospital in Bangladesh. J Natl Inst Neurosci Bangladesh, 2020;6(1): 33-37

Copyright: (C2020. Khanum et al. Published by Journal of National Institute of Neurosciences Bangladesh. This article is published under the Creative Commons CC BY-NC License (https://creativecommons.org/licenses/by-nc/4.0/). This license permits use, distribution and reproduction in any medium, provided the original work is properly cited, and is not used for commercial purposes.

\section{Introduction}

Morbid adherent placenta is an abnormal invasion of placental tissue (trophoblast) into outer or inner myometrium or through the serosa of the uterus termed as accreta, increta, percreta respectively. It is a potentially life threatening condition responsible for 7.0 
to $10.0 \%$ of maternal mortality ${ }^{2}$. Morbidly adherent placenta occur when there is partial or total absence of decidua basalis and Nitabuch layer.

The incidence of morbidly-adherent placenta which is previously thought to be very uncommon, is rising in contemporary obstetrical practice and obstetricians must be aware of this ${ }^{4}$. Massive obstetric haemorrhage is the principal clinical problem and is a potentially life threatening condition associated with high morbidity and mortality of up to $10.0 \%$ of patients ${ }^{5}$. Previously thought to be very rare, the incidence of placenta accraeta has increased ten-folds in the past 50 years. The two most important risk factors are an associated previa, a prior cesarean delivery, and more likely a combination of the two. Others risk factors are previous uterine surgery, previous dilatation and curettage, previous history of morbidly-adherent placenta previous myomectomy, ashermann Syndrome (endometrial defects), submucous leiomyoma, advanced maternal age, multiparity ${ }^{3}$.

Rising Caesarean section rate and short interval between caesarean section and conception is a major contributing factor $^{5}$. The exact aetiology is unknown; however, it has been postulated to the damage of decidua basalis which allows for placental invasion into myometrium. The barrier function of the decidua is absent in this condition, and the invasive trophoblast may invade the myometrium up to varying depths, from the most superficial (Placenta accrete) to deep myometrium (placenta increta) with breaching of uterine serosa (Placenta percraeta) and possibly invasion into adjacent organs ${ }^{7}$.

The layer of decidua normally separates the placental villi and myometrium at the site of placenta implantation. A placenta that directly adheres to the myometrium without an intervening decidual layer is termed placenta accrete. Furthermore according to the amount of placental involvement morbid adherent placenta is divided into three types like focal adherence where a single cotyledon is involved and partial adherence where one or several cotyledon are involved ${ }^{3}$. In total adherence the entire placenta is involved. Obstetric haemorrhage is one of the leading causes of maternal death in developing countries like Bangladesh. Abnormal placentation laccreta, increta, perereta has emerged over uterine atony as the leading indication for peripartum hysterectomy ${ }^{7}$. It is the most frequent indication for peripartum hysterectomy ${ }^{1}$. In addition, the incidence of perinatal complication is also increased due to preterm birth and small for gestation age fetuses. This present study was undertaken to identify the risk factors predisposing to morbidly adherent placenta, different modes of management and maternal and neonatal outcomes.

\section{Methodology}

This prospective cohort study was conducted in the Department of Obstetrics and Gynaecology in a tertiary care hospital in Savar named Enam Medical College, Dhaka, Bangladesh from January to December 2018 for a period of one (01) year. The study included all pregnant women, irrespective of parity status, with gestational age more than 29 weeks who had any type of morbid adherent placenta. The diagnosis was made only during attempted manual removal when the plane of cleavage between the placenta and the uterine wall could not be made out. Ultrasound imaging, color Doppler and MRI had all been valuable in the diagnosis of placenta accrete, increta and percreta during pregnancy. USG findings suggestive of placenta accrete was the loss of normal hypoechoic retroplacental myometrial zone, thinning and disrupting of the uterine serosa-bladder interface and focal exophytic masses invading the bladder. Color flow Doppler study showed hypervasculariry of serosa bladder interface. MRI reveals detour vessels and dark intraplacental bands on T2-weighted imaging. Unexplained rise of maternal serum aFP was observed with placenta accrete. Pathological confirmation included the absence of decidua basalis, absence of Nitabuch's fibrinoid layer and varying degree of penetration of the villi into the muscle bundles (increta) or up to the serosal layer (percreta). The risks included hemorrhage, shock, infection and rarely inversion of the uterus. All patients delivered in this institution. The complete history, physical examination of the patient along with relevant investigations for diagnosis of morbid adherent placenta were documented. The parameters studied were the mood of the presentation of the patient and duration of gestation, history of previous lower segment or classical caesarean section or other intrauterine operative procedure like dilatation and curettage, manual removal of placenta, myomectomy, mood of delivery of current pregnancy, by lateral uterine ligation, internal iliac artery ligation, hysterectomy-classical or lower segment or peri-partum, neonatal outcome and duration of the hospital stay.

\section{Results}

During the study period there were 1176 deliveries of which 15 patients had morbid adherent placenta, giving an incidence on 12.75 per 1000 pregnancies. Maximum number of patients were in the age group of 25 to 28 years which was $6(40 \%)$ cases followed by 33 to 38 years and 29 to 32 years of age group which were $5(33.0 \%)$ cases and $4(26.0 \%)$ cases respectively. 
Gravida 3 was in 9(60\%) cases; gravida second was in $20.0 \%$ patient and gravida 3 and above was about $20.0 \%$. Previous caesarean section was $10(66.0 \%)$, previous curettage $1(7.0 \%), 4(27.0 \%)$ patient was both caesarean section and curettage, Placenta praevia was 14(93.0\%). Placenta increta was 7(47.0\%), accrete $2(13.0 \%)$ and percreta $6(40.0 \%)$ (Table 1).

Table 1: Incidence, Demographic Profile And Risk Factors For Morbidly Adherent Placenta

\begin{tabular}{lcc}
\hline Demographic variable & Frequency & Percent \\
\hline Morbidly Adherent Placenta & 15 & \\
Age Group & & \\
- 25 to 28 years & 6 & 40.0 \\
- 29 to 32 years & 4 & 26.0 \\
- 33 to 38 years & 5 & 33.0 \\
Gravidity Group & & \\
- Gravida- 2 & 3 & 20.0 \\
- Gravida- 3 & 9 & 60.0 \\
- Gravida 3and above & 3 & 20.0 \\
Associated Risk Factors & & \\
- Previous Caesarean Section & 10 & 66.0 \\
- Previous Curettage & 1 & 7.0 \\
- Both & 4 & 27.0 \\
Location of placentation & & \\
- Placenta Praevia & 14 & 93.0 \\
- Normal Situated & 1 & 7.0 \\
Type of morbid adherent placenta & & \\
- Accreta & 2 & 13.0 \\
- Increta & 7 & 47.0 \\
- Percreta & 6 & 40.0 \\
Time of Diagnosis & & \\
- Antipartum & 10 & 67.0 \\
- Postpartum & 20.0 \\
- Intrapatum & & \\
\hline & & \\
\hline
\end{tabular}

Gestational age of the study population at the time of delivery were recored. Majority of the women were in 36 to 37 weeks of gestational age which was $7(47.0 \%)$ cases followed by 32 to 35 weeks of gestational age which were $4(27.0 \%)$ cases. Both in 29 to 31 weeks and more than 38 weeks of gestational age group were in $2(13.0 \%)$ cases each (Table 2$)$.

The treatment outcomes among the study popualtion were recorded. Hysterectomy without other procedure was performed in 5(33.0\%) cases. Hysterectomy with other procedure was performed in $2(13.0 \%)$ cases. Other procedure without hysterectomy was done in $6(40.0 \%)$ cases. No other procedure or hysterectomy was done in $2(13.0 \%)$ cases (Table 3$)$.
Table 2: Gestational Age of the Study Population at The time of Delivery

\begin{tabular}{lcc}
\hline Gestational Age & Frequency & Percent \\
\hline 29 to 31 weeks & 2 & 13.0 \\
32 to 35 weeks & 4 & 27.0 \\
36 to 37 weeks & 7 & 47.0 \\
>38 weeks & 2 & 13.0 \\
Total & $\mathbf{1 5}$ & $\mathbf{1 0 0 . 0}$ \\
\hline
\end{tabular}

Table 3: Treatment Outcomes among the Study Popualtion

\begin{tabular}{lcc}
\hline Treatment Modality & Frequency & Percent \\
\hline Hysterectomy without other procedure & 5 & 33.0 \\
Hysterectomy with other procedure & $2^{*}$ & 13.0 \\
Other procedure without hysterectomy & $6^{*}$ & 40.0 \\
No other procedure no hysterectomy & $2^{* *}$ & 13.0 \\
\hline
\end{tabular}

* BLUAL,B-Lynch suture; ** methotrexate-4

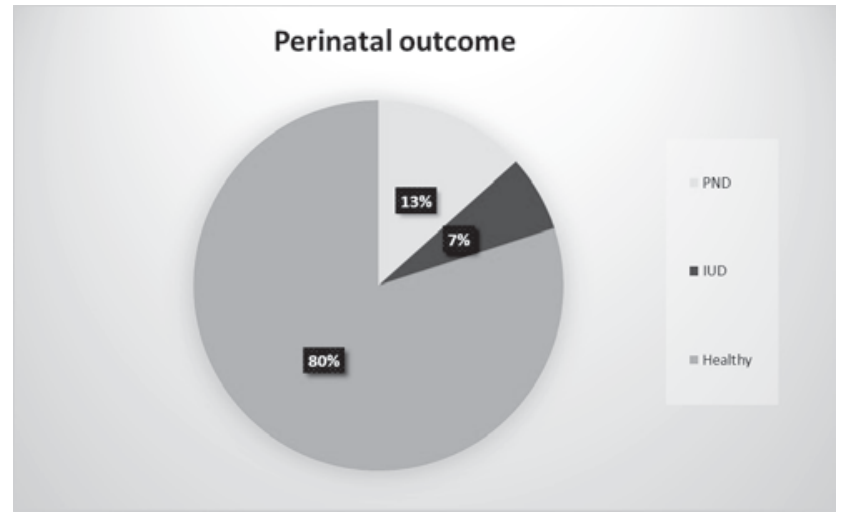

Figure I: Fetal Outcomes among the Study Population

Fetal outcomes were recorded. In this study PND was found in $13.0 \%$ cases. IUD was reported in $7.0 \%$ cases. However $80.0 \%$ cases were found healthy (Figure I).

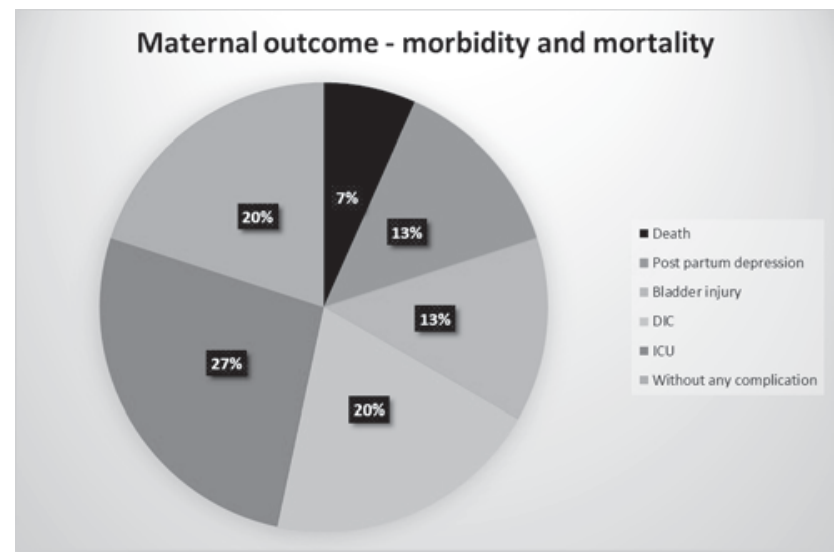

Figure II: Maternal outcome among the Study Population 
Maternal outcomes were recorded. In this study DIC was found in $20.0 \%$ cases. ICU admission was needed in $27.0 \%$ cases. However $13.0 \%$ cases were reported with bladder injury. Post-partum depression was reported in $13.0 \%$. Furthermore, death was reported in $7.0 \%$ cases. No complication was found in $20.0 \%$ cases (Figure II).

\section{Discussion}

Diagnosis of morbid adherent placenta helps in saving maternal life. This helps in counseling and monitoring of high risk patients for morbid adherent placenta on the basis of history and ultrasonography ${ }^{8}$. Clinical suspicion should be raised in all women with vaginal bleeding after 20 weeks of gestation. The definitive diagnosis of most low-lying placentas is now achieved with ultrasound imaging. The UK National Screening Committee supports practice of identifying at the routine 20-week antenatal screening ultrasound scan of women whose placenta encroaches on the cervical os?. Successful management of this condition requires antenatal diagnosis and referral to a tertiary care centre where multidisciplinary expertise, blood transfusion facilities and intensive care units are available ${ }^{10}$. Therefore this present study has been performed in a referral tertiary care hospital which represents real scenario. It is also $=$ very important that all the caesarean sections performed in women with placenta previa and previous section should be conducted by skilled and experienced obstetrician ${ }^{11}$.

Traditional management is cesarean hysterectomy which has reduced the morbidity and mortality ${ }^{8}$. Hysterectomy is favorable method for accrete and increta where there is no extra-uterine invasion with delivery of foetus through classical, fundal, or high transverse incision avoiding incision of placenta ${ }^{6}$. The placenta may either be removed or left attached to uterus and removed as a part of hysterectomy. The placenta attached with uterus followed by hysterectomy reduces significantly blood loss and morbidity and mortality. Hysterectomy although lifesaving if timely attempted but the resultant loss of fertility is devastating if patient is young. Secondly morbidity is high if it is percreta. For this reason, conservative approach has been proposed ${ }^{11-13}$.

In this study 1176 deliveries are performed of which 15 patients have morbid adherent placenta, giving an incidence on 12.75 per 1000 pregnancies. Similar result has been reported by different studies. In a study ${ }^{14}$ frequency of morbidly-adherent placenta is found in $1 / 3104$. It has been estimated that the incidence of placenta accreta was approximately 1 in 4027 in 1970s, 1 in 2510 in the 1980 s, 1 in 533 pregnancies in 19822002 and 1 in 210 in $2006^{2}$.

In this study there are several risk factors noted. Among these previous caesarean section is $10(66.0 \%)$ and previous curettage 1(7.0\%); however, $4(27.0 \%)$ patients are presented with both caesarean section and curettage. Placenta praevia is reported in $14(93.0 \%)$ cases. Placenta increta is $7(47.0 \%)$ cases, accrete in $2(13.0 \%)$ cases and percreta in $6(40.0 \%)$ cases. Marked increased in incidence of morbidly-adherent placenta is due to increased rate of caesarean sections. At present, frequency has increased one per 2,500 to one per 110 deliveries $^{6}$. It establishes that morbid adherent placenta is directly related with frequency of caesarean section. Every effort should be made to reduce percent of primary caesarean section so that morbidity and mortality related to morbid adherent placenta can be reduced $^{11}$. If cesarean rates continue to increase, the annual incidence of placenta previa, placenta accreta, and maternal death will also rise substantially.

Maximum number of patients were in the age group of 25 to 28 years which was $6(40 \%)$ cases followed by 33 to 38 years and 29 to 32 years of age group which were $5(33.0 \%)$ cases and 4(26.0\%) cases respectively. Gravida 3 was in $9(60 \%)$ cases; gravida second was in $20.0 \%$ patient and gravida 3 and above was about $20.0 \%$. Gestational age of the study population at the time of delivery were recored. Majority of the women were in 36 to 37 weeks of gestational age which was $7(47.0 \%)$ cases followed by 32 to 35 weeks of gestational age which were $4(27.0 \%)$ cases. Both in 29 to 31 weeks and more than 38 weeks of gestational age group were in $2(13.0 \%)$ cases each. The treatment outcomes among the study popualtion were recorded. Hysterectomy without other procedure was performed in $5(33.0 \%)$ cases. Hysterectomy with other procedure was performed in $2(13.0 \%)$ cases. Other procedure without hysterectomy was done in $6(40.0 \%)$ cases. No other procedure or hysterectomy was done in $2(13.0 \%)$ cases. It has been established that the conservative management involves leaving placenta in situ and this may be complemented by bilateral embolization of uterine arteries, parenteral methotrexate or both ${ }^{12}$. Balloon occlusive devices can be placed in both internal iliac arteries before surgery by an interventional radiologist. Conservative management of placenta accreta and increta is now an acceptable and reliable alternative to radicle surgery ${ }^{13}$. This case series clearly describes the high morbidity associated with undiagnosed adherent placentas in antenatal period 
resulting in massive haemorrhage due to piecemeal removal of placenta, whereas in diagnosed cases placenta have left attached to uterus.

Fetal outcomes were recorded. In this study PND was found in $13.0 \%$ cases. IUD was reported in $7.0 \%$ cases. However $80.0 \%$ cases were found healthy. The main newborn complication was prematurity and the average gestational age in our study was 32.1 weeks. $55 \%$ of the newborns were preterm with an average birth weight low birth weight. Maternal outcomes are recorded. In this study DIC is found in $20.0 \%$ cases. ICU admission is needed in $27.0 \%$ cases. However $13.0 \%$ cases are reported with bladder injury. Post-partum depression is reported in $13.0 \%$. Furthermore, death is reported in $7.0 \%$ cases. No complication is found in $20.0 \%$ cases. In a study it has been reported that the maternal mortality is $10.0 \%$, which is comparable to the rate of 7 to $10.0 \%$ as quoted in literature ${ }^{12}$. The biggest risk with accreta is severe bleeding because the placenta cannot detach properly at birth. In the short term, this can require multiple blood transfusions, cause a life-threatening blood clotting crisis, or necessitate a hysterectomy ${ }^{14}$. In the long term, it can cause postpartum anemia, difficulty breastfeeding, or even Sheehan's Syndrome (damage to the mother's pituitary gland, resulting in long-term health problems). Since major blood loss is the biggest risk of an accreta treatment of accretas usually revolves around trying to proactively prevent as much blood loss as possible ${ }^{9}$. Correction of anaemia prior to expected blood loss. Blood bank preparations include arrangement of cross matched blood and component therapy. It will help to reduce maternal morbidity and mortality.

\section{Conclusion}

In conclusion young adult women are mostly suffereing from morbidly-adherent placenta. Most of the patients of this study have given the history of previous caesarean section. It has been also found that the co-existenting of placenta praevia patients are also commonly suffering from morbidly adherent placenta. Further large scale multicenter study should be conducted.

\section{References}

1. Chaudhari HK, Shah PK, D'Souza N. Morbidly adherent placenta: Its management and maternal and perinatal outcome. The Journal of Obstetrics and Gynecology of India. 2017;67(1):42-7

2. Bajwa SK, Singh ABS. Contemporary issues in the management of abnormal placentation during pregnancy in developing nations: an Indian perspective. Int J Crit Illn Inj Sci. 2013;3(3):183-189

3. Bajwa SK, Singh A, Bajwa SJ. Contemporary issues in the management of abnormal placentation during pregnancy in developing nations: An Indian perspective. International journal of critical illness and injury science. $2013 \mathrm{Jul} ; 3(3): 183$

4. Bauer ST, Bonanno C. Abnormal placentation. InSeminars in perinatology 2009 Apr 1 (Vol. 33, No. 2, pp. 88-96). WB Saunders. 5. Yi KW, Oh M-J, Seo T-S, et al. Prophylactic hypogastric artery ballooning in a patient with complete placenta previa and increta. $\mathrm{J}$ Korean Med Sci. 2010;25(4):651-655

6. Roethlisberger M, Womastek I, Posch M, et al. Early postpartum hysterectomy: incidence and risk factors. Acta Obs Gynecol Scand. 2010;89(8):1040-1044

7. Eller AG, Porter TF, Soisson P, et al. Optimal management strategies for placenta accreta. BJOG. 2009;116(5):648-654

8. Sumigama $\mathrm{S}$, Itakura $\mathrm{A}$, Ota $\mathrm{T}$, et al. Placenta previa increta/percreta in Japan: a retrospective study of ultrasound findings, management and clinical course. J Obstet Gynaecol Res. 2007;33(5):606-611

9. Tikkanen M, Paavonen J, Loukovaara MSV. Antenatal diagnosis of placenta accreta leads to reduced blood loss. Acta Obs Gynecol Scand. 2011;90(10):1140-1146

10. Bennett MJSR. "Conservative" management of placenta praevia percreta: report of two cases and discussion of current management options. Aust N Z J Obs Gynaecol. 2003;43:249-251 11. Aggarwal R, Suneja A, Vaid NB, et al. Morbidly adherent placenta: a critical review. J Obstet Gynaecol India. 2012;62(1):57-61

12. Wu S, Kocherginsky MHJ. Abnormal placentation: twentyyear analysis. Am J Obs Gynecol. 2005;192(5):1458-1461

13. Garmi G, Salim R. Epidemiology, etiology, diagnosis, and management of placenta accreta. Obstet Gynecol Int. 2012;2012:873929

14. Herath R, Wijesinghe P. Management of morbidly adherent placenta. Sri Lanka J Obstet Gynaecol. 2012;33(2):39-44 